\title{
Clinical characteristics and respiratory support of 310 COVID-19 patients, diagnosed at the emergency room: a single-center retrospective study
}

\author{
Sandro Luigi Di Domenico ${ }^{1}$ (1) . Daniele Coen ${ }^{1} \cdot$ Marta Bergamaschi $^{2} \cdot$ Valentina Albertini $^{2} \cdot$ Leonardo Ghezzi $^{1}$. \\ Michela Maria Cazzaniga ${ }^{1}$ - Valeria Tombini ${ }^{1} \cdot$ Riccardo Colombo $^{1} \cdot$ Nicolò Capsoni $^{2} \cdot$ Tommaso Coen $^{3}$. \\ Katia Barbara Cazzola ${ }^{1} \cdot$ Marina Di Fiore $^{1} \cdot$ Laura Angaroni $^{1} \cdot$ Marco Alberto Strozzi $^{1}$
}

Received: 5 May 2020 / Accepted: 21 October 2020 / Published online: 11 November 2020

c) Società Italiana di Medicina Interna (SIMI) 2020

\begin{abstract}
An ongoing outbreak of pneumonia associated with severe acute respiratory coronavirus 2 (SARS-CoV-2) occurred at the end of February 2020 in Lombardy, Italy. We analyzed data from a retrospective, single-center case series of 310 consecutive patients, with confirmed SARS-CoV-2 infection, admitted to the emergency room. We aimed to describe the clinical course, treatment and outcome of a cohort of patients with COVID-19 pneumonia, with special attention to oxygen delivery and ventilator support. Throughout the study period, 310 consecutive patients, with confirmed SARS-CoV-2 infection, attended the Emergency Room (ER), of these, 34 were discharged home directly from the ER. Of the remaining 276 patients, the overall mortality was 30.4\%: 7 patients died in the ER and 77 during hospitalization. With respect to oxygen delivery: 22 patients did not need any oxygen support (8.0\%), 151 patients were treated with oxygen only (54.7\%), and $49(17.8 \%)$ were intubated. 90 patients (32.6\%) were treated with CPAP (Continuous Positive Airway Pressure) or NIV (Non Invasive Ventilation); in this group, 27 patients had a Do Not Intubate (DNI) order and were treated with CPAP/NIV as an upper threshold therapy, showing high mortality rate (88.9\%). Among the 63 patients treated with CPAP/NIV without DNI, NIV failure occurred in 36 patients $(57.1 \%)$, with mortality rate of $47.2 \%$. Twenty-seven (27) patients were treated with CPAP/NIV without needing mechanical ventilation and 26 were discharged alive (96.3\%). The study documents the poor prognosis of patients with severe respiratory failure, although a considerable minority of patients treated with CPAP/NIV had a positive outcome.
\end{abstract}

Keywords SARS-CoV-2 infection · COVID-19 $\cdot$ CPAP (continuous positive airway pressure) $\cdot$ NIV (non-invasive ventilation) $\cdot$ Epidemiology

\section{Introduction}

On February 21, 2020, the first person-to-person transmission of SARS-CoV-2 in Italy was reported, leading to one of the largest COVID-19 outbreaks, causing more than 35,000

Sandro Luigi Di Domenico

didomenico.sandro@gmail.com

1 Department of Emergency Medicine, ASST Grande Ospedale Metropolitano Niguarda, Piazza Ospedale Maggiore 3, 20162 Milan, Italy

2 Università Degli Studi Milano Bicocca, Piazza dell'Ateneo Nuovo 1; 20126, Milan, Italy

3 Department of Economics, Brown University, Providence, RI, USA deaths. Epidemiological data from Italy are somehow at variance with those that have been reported for China and other countries. In particular, higher mortality rates have been described [1] This evidence has been attributed to various possible causes, among which, it is important to emphasize the different age distribution of the population, the different prevalence of comorbid conditions and a more conservative policy for carrying on diagnostic swabs.

Although much epidemiological information is currently available [2-4], detailed clinical data are still scarce and mainly refer to the subset of patients admitted to intensive care units [5-7]. Much less is known about the clinical course of the patients who access the Emergency Room (ER) for fever or respiratory symptoms of differing severity. Moreover, there is a big variability in regard to the use of continuous positive air pressure (CPAP) and bilevel 
non-invasive ventilation (NIV) and there is a lack of data about clinical course and outcome of patients treated with these supports $[2,5,8]$.

This study retrospectively analyzed data from a single center in Milan, Italy, aiming to describe the characteristics and outcome of patients accessing the ER for COVID-19. We also explored the potential association between a number of clinical variables and mortality, with particular attention to the use of CPAP and NIV for respiratory support.

\section{Methods}

\section{Study participants}

This retrospective study was conducted in a single hospital: Grande Ospedale Metropolitano Niguarda in Milan (900 beds). We collected data from patients admitted to the Emergency Room (ER), from February 29 to March 19, 2020. Three hundred and ten consecutive patients with confirmed SARS-Cov-2 infection were enrolled in the study. According to the WHO guidance [9], laboratory confirmation for SARS-Cov-2 was defined as a positive result of real-time reverse transcriptase-polymerase chain reaction (RT-PCR) assay of nasal and pharyngeal swabs [10]. Figure 1 shows the recruitment flowchart.

The study was approved by the institutional review board at Grande Ospedale Metropolitano Niguarda. Written informed consent was waived by the ethics commission of the designated hospital, in accordance with governmental regulations on observational retrospective studies.

\section{Data collection}

The demographic characteristics (age and sex) and clinical data (symptoms, symptoms onset, comorbidities, home treatments) were collected from electronic medical records. Diagnostic work-up in the ER (laboratory test, ultrasound and radiologic findings) were similarly collected. Patients' treatment (drugs, respiratory support) and outcome were followed and recorded throughout hospital stay. Causes of death were retrieved from certificates of death. Some data were missing due to the incomplete fulfillment of clinical notes or the clinical decision of not performing a particular test.

\section{Criteria for hospital admission and respiratory support}

Criteria for admission were not strictly defined, but physicians working in the ED behaved uniformly. Patients with oxygen saturation above $93 \%$ breathing in ambient air, no signs of respiratory distress and good general conditions were generally sent home after $6 \mathrm{~h}$ of observation. However, patients with older age, multiple comorbidities and marked radiological or biochemical alterations, were usually admitted. Patients with intolerable symptoms such as fever, fatigue, diarrhea or headache, were similarly admitted.

Patients discharged home were asked to self-isolate from the rest of their co-habitants, using a single room, and if possible a private bathroom. They were given written advice and they were reported to local health authorities for quarantine and follow-up. Patients discharged home were followed up on electronic medical record and they were called by phone to check their condition after one month from the discharge. The impossibility of staying in a proper quarantine, together with social frailty or a language barrier, were considered criteria for admission.

Patients with SpO2 90-94\% were given oxygen up to $12 \mathrm{~L} / \mathrm{min}$, aiming at values of $\mathrm{SpO} 2>94 \%$. Patients with $\mathrm{SpO} 2<90 \%$, or $90-94 \%$ while on oxygen up to $12 \mathrm{~L} / \mathrm{min}$, were treated with CPAP or NIV. Patients whose SpO2 was $<90 \%$ with oxygen $12 \mathrm{~L} / \mathrm{min}$ or CPAP/NIV, and those with arterial oxygen partial tension/fraction inspired oxygen $(\mathrm{PaO} 2 / \mathrm{FiO} 2)<200 \mathrm{mmHg}$ or persistent respiratory fatigue, were considered for immediate intubation unless a do-not-intubate (DNI) order had been issued. DNI was issued by an expert anesthesiologist in agreement with the emergency physician, after considering the severity of the disease, patients age and comorbidities. In case of disagreement, a second opinion by a senior anesthesiologist was obtained.

Patients with COVID-19 infection were admitted to medical wards. Patients treated with CPAP/NIV were admitted to Emergency Medicine or Respiratory Medicine wards. Patients needing mechanical ventilation were admitted directly to the Intensive Care Unit (ICU) or transferred from non-intensive wards, in case of clinical deterioration or CPAP/NIV failure.

\section{Statistical analysis}

Descriptive statistics were obtained for all study variables. Continuous variables are presented as median and interquartile range (IQR) or as mean and standard error (SE). Categorical data are expressed as proportions. The prognostic relevance of possible risk factors is evaluated with a Cox proportional hazards model. The $t$ test has been used to determine if the means of two sets of data are significantly different from each other. 


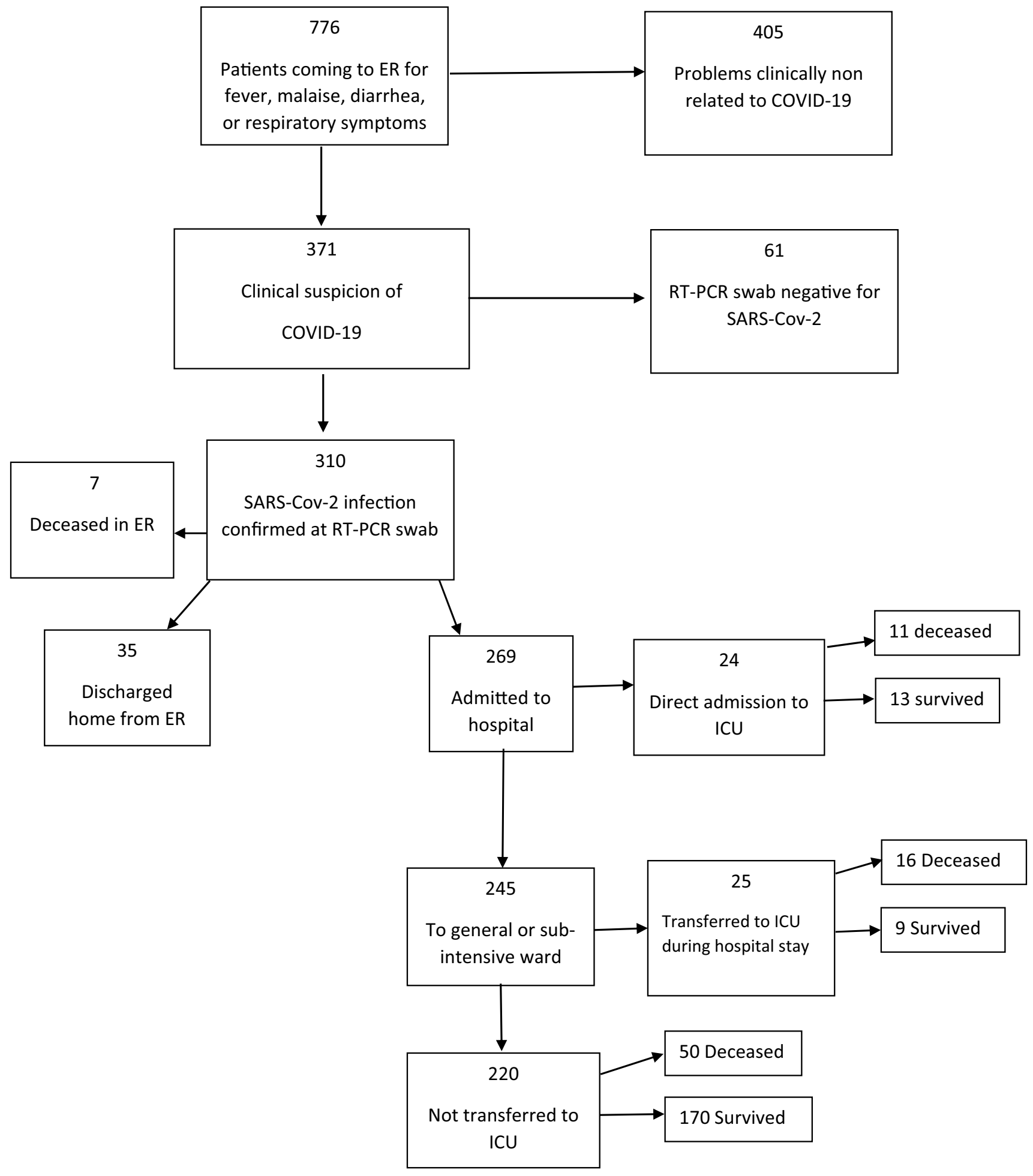

Fig. 1 Flowchart of patients recruitment and outcome 


\section{Results}

\section{Patient characteristics}

Throughout the period of study, 776 patients attended the ER with fever, diarrhea, headache, syncope or respiratory symptoms; 405 had no disease related to COVID-19. Of the 371 remaining, 61 were excluded because of missing or negative real time-PCR from a nasopharyngeal swab. 34 patients were discharged home from the ER, 7 died in the ER and 269 were admitted to hospital. Three patients were admitted because of the impossibility of self-isolation for proper quarantine or because of social frailty. Patients discharged home from the ER were younger than those admitted to hospital (median 46 [IQR 37.53] vs. 67 [IQR: 54.77]) and had fewer comorbidities (median 0 [IQR 0.2 ] vs. 1 [IQR 0.2]). During the study period, only 5 of the patients sent home from the ER came back to hospital and 2 of them were subsequently admitted to medical ward. None of the re-admitted patients needed oxygen support and no death was recorded. Two patients were lost in the follow-up because of wrong or missing telephone number. With respect to the patients admitted, 84 (30.4\%) died in the hospital and 192 (69.6\%) were discharged home. Death rate in the ICU was $55.1 \%$ (27 patients out of 49).

Patients aged 18-60 had lower mortality (10.2\%), compared to patients aged between 61 and 75 years (38.6\%) and $>75$ years $(48.8 \%)$ Table 1.

The median age of patients was 64 years overall (IQR 52-76), 46 years for those discharged from the ER (IQR 37-53) and 67 for those admitted to the hospital (IQR 54-77). Females were 110 (35.5\%). Univariate and multivariate Cox regression models were used to evaluate associations of covariates with risk of death. Considering the total number of deaths $(n=84)$ in our study, 8 covariates were chosen for multivariate Cox model on the basis of univariate analysis $(P<: 05)$. The presence of any chronic illnesses (hypertension, diabetes, heart disease, lung disease, cancer) was associated with an increase in mortality. The association with mortality remained significant at the $5 \%$ level for most covariates in the multivariable setting Fig. 2; Table 2.

Eighty-nine patients were under chronic medication with angiotensin-converting enzyme inhibitors (ACEI) or angiotensin receptor blockers (ARB); after controlling for age and diagnosis of hypertension, we found no significant association between mortality and chronic use of ACEI or ARB drugs. On the contrary, regressing mortality on CRP at admission we find that the association is significant at any conventional level (an increase in CRP of $1 \mathrm{mg}$ / $\mathrm{dL}$ is associated with an increase in mortality of $1.8 \%$ ).
Mortality is $10.9 \%$ for the 119 patients with CRP $<3 \mathrm{mg}$ / dL (SE: 0.029$), 31.1 \%$ for the 119 patients with a CRP $3-10 \mathrm{mg} / \mathrm{dL}$ (SE: 0.043 ) and $47.2 \%$ for the 72 patients with a CRP $>10 \mathrm{mg} / \mathrm{dL}$ (SE: 0.059). Chest X-ray was used initially as the first imaging exam, but it was progressively abandoned in favor of lung ultrasound and/or CT scan. Chest X-ray showed low concordance (76\%) compared to CT scan, which is considered the gold standard for detecting COVID-19 Pneumonia. On the other hand bedside lung ultrasonography showed a concordance of $95.0 \%$ when compared with chest CT scan.

Twenty-two patients did not need any oxygen support. In this group, no death was recorded. Mortality was $21.2 \%$ among the 151 patients treated with oxygen only. Ninety (90) patients were treated with CPAP/NIV: 48 survived (53\%) and were discharged, 42 died (47\%). Twentyseven (27) patients had a do-not-intubate (DNI) order and were treated with CPAP/NIV as an upper threshold therapy, showing high mortality rate (24 deaths out of 27 patients: mortality rate $88.9 \%$ ). Among the 63 patients, treated with CPAP/NIV, without DNI, CPAP/NIV failure occurred in 36 patients $(57.1 \%)$, with mortality rate of $47.2 \%$ (17 patients deceased and 19 survived). The most of the patients (29 patients out of the 36) who had first been assigned to CPAP/NIV, underwent endotracheal intubation (ETI) on the same day or the day after starting noninvasive ventilation. Twenty-seven patients were treated with CPAP/NIV, without needing mechanical ventilation; achieving survival in 26 patients (96.3\%). Thirteen (13) patients were intubated on arrival in ER or in the prehospital setting, without any trial of CPAP/NIV. Mortality in this group was 76.9\% (10 patients out of 13) (Table 3).

All the deceased patients died because of respiratory failure secondary to COVID-19 pneumonia and ARDS. Clinical course was complicated by septic shock or bacterial super-infection in 8 patients (4 in the ETI group, 2 in the NIV failure/ETI group, 1 case of Legionella pneumonia in the NIV/DNI group and 1 case of methicillinresistant Staphylococcus aureus in the oxygen only group), multi-organ failure (MOF) in 9 patients (4 in the ETI group, 4 in the NIV failure/ETI group and 1 in the oxygen only group), 1 case of pulmonary embolism in the NIV/ DNI group and 1 case of ventilator-induced lung injury (VILI) in the NIV failure/ETI group.

Table 4 shows baseline characteristics of patients treated with invasive and non-invasive ventilation. Patients treated with CPAP/NIV only were characterized by younger age (mean 58.4 [SE 2.78] vs. 76.4 [SE 1.37] years), less number of comorbidities and a less severe degree of respiratory insufficiency (mean $\mathrm{PaO} 2 / \mathrm{FiO} 2248$ [SE 16.7] vs. 186 [SE 19.6]), compared with patients who received a DNI order. Patients treated with CPAP/NIV only had also higher $\mathrm{PaO} 2 / \mathrm{FiO} 2$ than those intubated, but 
Table 1 Clinical, biochemical and radiological characteristics of patients with COVID-19

\begin{tabular}{|c|c|c|c|}
\hline Variable & Number & Percentage (IQR) & Observations \\
\hline \multicolumn{4}{|l|}{ Demography } \\
\hline Patients & 310 & 100 & 310 \\
\hline Age, median & 64 & $(52-76)$ & 310 \\
\hline Female & 110 & 35.5 & 310 \\
\hline \multicolumn{4}{|l|}{ Signs and symptoms at admission } \\
\hline Fever & 288 & 92.9 & 310 \\
\hline Cough & 183 & 59.2 & 309 \\
\hline Shortness of breath & 107 & 34.5 & 310 \\
\hline Rhinorrhea or conjunctivitis & 8 & 2.62 & 305 \\
\hline Anosmia, dysgeusia & 43 & 14.1 & 304 \\
\hline Fatigue or muscle ache & 5 & 1.64 & 304 \\
\hline Headache & 32 & 10.4 & 307 \\
\hline Gastrointestinal symptoms & 40 & 13.2 & 304 \\
\hline \multicolumn{4}{|l|}{ Comorbidities } \\
\hline Smoke & 11 & 7.28 & 151 \\
\hline Obesity (variable) & 49 & 22.9 & 214 \\
\hline Obesity (BMI > 30) & 34 & 32.7 & 104 \\
\hline Hypertension & 134 & 43.5 & 308 \\
\hline Diabetes & 53 & 17.2 & 308 \\
\hline Heart disease & 50 & 16.2 & 309 \\
\hline Vascular disease & 39 & 12.7 & 308 \\
\hline Chronic obstructive pulmonary disease & 42 & 13.5 & 310 \\
\hline Immunosuppression or rheumatoid arthritis & 20 & 6.47 & 309 \\
\hline Cancer & 27 & 8.77 & 308 \\
\hline \multicolumn{4}{|l|}{ Laboratory findings at admission, median (IQR) } \\
\hline Lymphocytes/L & 1.03 & {$[0.74,1.39]$} & 283 \\
\hline $\mathrm{CPR}, \mathrm{mg} / \mathrm{dL}$ & 4.6 & {$[1.55,9.75]$} & 308 \\
\hline Creatinine, $\mathrm{mg} / \mathrm{dL}$ & 0.99 & {$[0.82,1.2]$} & 298 \\
\hline Bilirubin, mg/dL & 0.5 & {$[0.36, .7]$} & 287 \\
\hline INR & 1.13 & {$[1.07,1.21]$} & 248 \\
\hline aPTT & 1.1 & {$[0.99,1.26]$} & 247 \\
\hline $\mathrm{pH}$ & 7.46 & {$[7.44,7.49]$} & 239 \\
\hline $\mathrm{pO} 2, \mathrm{mmHg}$ & 69 & {$[59.5,79]$} & 240 \\
\hline $\mathrm{pCO} 2, \mathrm{mmHg}$ & 32 & {$[29,35]$} & 241 \\
\hline $\mathrm{PaO} 2 / \mathrm{FiO} 2$ & 300 & {$[233,347]$} & 235 \\
\hline \multicolumn{4}{|l|}{ Chest radiology } \\
\hline Echography, positive & 169 & 54.7 & 309 \\
\hline $\mathrm{X}$-rays, positive & 84 & 27.6 & 304 \\
\hline CT, positive & 257 & 83.2 & 309 \\
\hline \multicolumn{4}{|l|}{ Home therapy } \\
\hline ACEI & 55 & 17.9 & 307 \\
\hline $\mathrm{ARB}$ & 39 & 12.7 & 307 \\
\hline Other & 145 & 46.8 & 310 \\
\hline
\end{tabular}

$B M I$ body mass index, $C P R$ C-reactive protein, INR international normalized ratio, $a P T T$ activated prothrombin time, $\mathrm{PaO} 2 / \mathrm{FiO} 2$ arterial oxygen partial tension/fraction inspired oxygen, $C T$ computed tomography, $A C E I$ angiotensin-converting enzyme inhibitors, $A R B$ angiotensin II receptor blocker 
Fig. 2 Estimated coefficients and $95 \%$ confidence intervals for univariate and multivariate Cox regressions of patient survival on potential risk factors. $C K D$ chronic kidney disease
Table 2 Risk factors and death estimated with univariate and multivariate Cox regression models

\begin{tabular}{lllll}
\hline & Univariate HR $(95 \% \mathrm{CI})$ & $P$ value & Multivariate HR $(95 \%$ CI) & $P$ value \\
\hline Age (decades) & $1.744[1.386,2.194]$ & 0.000 & $1.540[1.159,2.047]$ & 0.003 \\
Diabetes & $3.507[1.983,6.201]$ & 0.000 & $1.912[1.030,3.547]$ & 0.040 \\
Hypertension & $2.967[1.652,5.329]$ & 0.000 & $1.201[0.558,2.585]$ & 0.640 \\
Heart disease & $3.684[1.879,7.221]$ & 0.000 & $1.921[0.893,4.135]$ & 0.095 \\
CKD & $4.604[2.153,9.844]$ & 0.000 & $3.861[1.765,8.446]$ & 0.001 \\
Obesity & $2.299[1.301,4.063]$ & 0.004 & $1.981[1.046,3.750]$ & 0.036 \\
Vascular disease & $2.860[1.338,6.113]$ & 0.007 & $1.008[0.329,3.086]$ & 0.989 \\
Ever been a smoker & $2.627[1.234,5.592]$ & 0.012 & $2.544[1.233,5.251]$ & 0.012 \\
Cancer & $2.418[0.965,6.059]$ & 0.060 & & \\
Pulmonary disease & $1.748[0.802,3.807]$ & 0.160 & & \\
\hline
\end{tabular}

Multivariate analysis has not been carried out for cancer and pulmonary disease, because univariate showed no significance

$H R$ hazard ratio, $C K D$ chronic kidney disease
Table 3 Oxygen support and outcome

\begin{tabular}{lrcc}
\hline Oxygen delivery & Number & Outcome \\
\cline { 3 - 4 } & & Survived (\%) & Deceased (\%) \\
\hline No need of oxygen & 22 & $22(100)$ & $0(0)$ \\
Oxygen only & 151 & $119(78.8)$ & $32(21.2)$ \\
CPAP/NIV only & 27 & $26(96.3)$ & $1(3.7)$ \\
DNI CPAP/NIV & 27 & $3(11.1)$ & $24(88.9)$ \\
NIV failure and ETI & 36 & $19(52.8)$ & $17(47.2)$ \\
ETI & 13 & $3(23.1)$ & $10(76.9)$ \\
Total & 276 & $192(69.6)$ & $84(30.4)$ \\
\hline
\end{tabular}

$C P A P / N I V$ continuous positive airway pressure/non-invasive ventilation, $D N I$ do-not-intubate order, ETI endotracheal intubation

\section{Hazard Rates (Cox regression)}

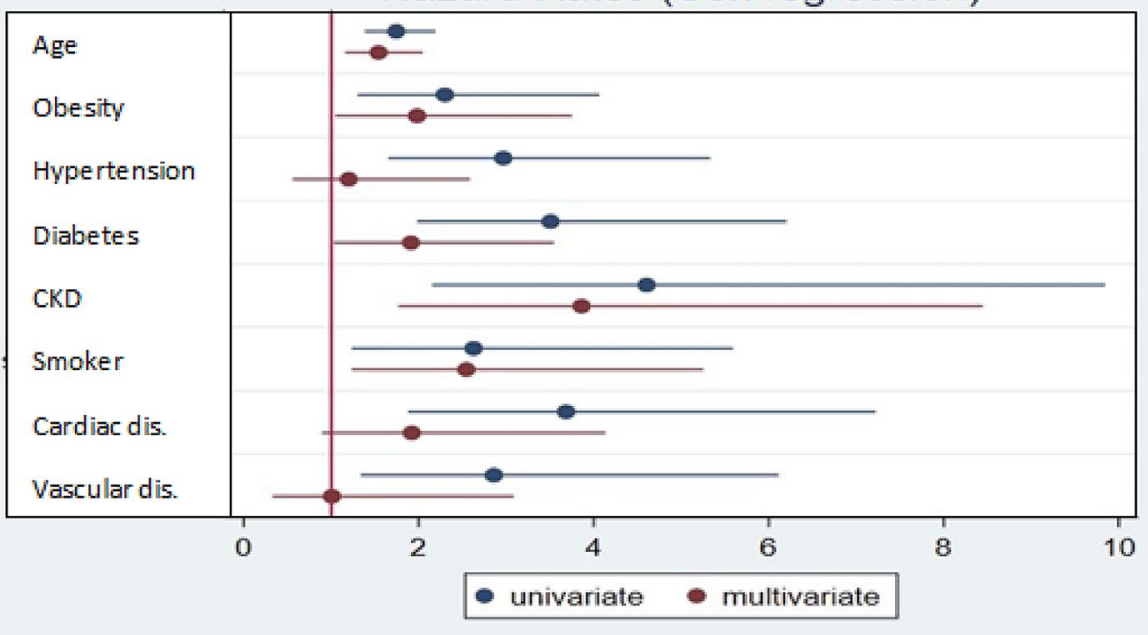


Table 4 Clinical and laboratory characteristics among patients treated with invasive and non-invasive ventilation

\begin{tabular}{|c|c|c|c|c|c|c|c|c|c|c|c|}
\hline Variable & $N$ & $\begin{array}{l}\text { (1) } \\
\text { CPAP/NIV only } \\
\text { Mean [SE] }\end{array}$ & $N$ & $\begin{array}{l}(2) \\
\text { DNI/NIV } \\
\text { Mean [SE] }\end{array}$ & $N$ & $\begin{array}{l}\text { (3) } \\
\text { ETI } \\
\text { Mean [SE] }\end{array}$ & $N$ & $\begin{array}{l}\text { (4) } \\
\text { NIV failure/ETI } \\
\text { Mean [SE] }\end{array}$ & $\begin{array}{l}t \text { test } \\
P \text { value } \\
(1)-(2)\end{array}$ & $\begin{array}{l}t \text { test } \\
P \text { value } \\
(1)-(3)\end{array}$ & $\begin{array}{l}t \text { test } \\
P \text { value } \\
(1)-(4)\end{array}$ \\
\hline ge & 27 & 58.444 [2.799] & 27 & 76.444 [1.377] & 13 & $56.231[3.444]$ & 36 & 61.222 [1.909] & $0.000 * * *$ & 0.640 & 0.400 \\
\hline Female & 27 & $0.259[0.086]$ & 27 & $0.111[0.062]$ & 13 & $0.154[0.104]$ & 36 & $0.222[0.070]$ & 0.167 & 0.467 & 0.738 \\
\hline Obesity & 24 & $0.292[0.095]$ & 15 & $0.267[0.118]$ & 11 & $0.545[0.157]$ & 29 & 0.414 [0.093] & 0.870 & 0.158 & 0.366 \\
\hline Hypertension & 27 & $0.370[0.095]$ & 26 & $0.731[0.0$ & 13 & $0.462[0.144]$ & 36 & $0.583[0.0$ & $0.008 * * *$ & 0.593 & $0.097 *$ \\
\hline Diabetes & 27 & $0.074[0.051]$ & 26 & $0.346[0.095]$ & 13 & 0.308 [0.133] & 36 & $0.222[0.070]$ & $0.014 * *$ & $0.054 *$ & 0.115 \\
\hline Heart disease & 27 & $.148[0.070]$ & 26 & $0.500[0.100]$ & 13 & $0.154[0.104]$ & 36 & $0.083[0.047]$ & $0.005 * * *$ & 0.963 & 0.426 \\
\hline Vascular disease & 27 & $0.037[0.037]$ & 26 & $0.231[0.084]$ & 13 & $0.077[0.077]$ & 36 & $0.056[0.039]$ & $0.038 * *$ & 0.599 & 0.738 \\
\hline COPD & 27 & $0.000[0.000]$ & 27 & $0.222[0.082]$ & 13 & $0.077[0.077]$ & 36 & $0.111[0.053]$ & $0.009 * * *$ & 0.152 & $0.076^{*}$ \\
\hline Immunosuppression & 27 & $0.074[0.051]$ & 27 & $0.111[0.062]$ & 13 & $0.000[0.000]$ & 36 & $0.056[0.039]$ & 0.646 & 0.327 & 0.770 \\
\hline Cancer & 27 & $0.000[0.000]$ & 26 & $0.192[0.079]$ & 13 & $0.000[0.000]$ & 36 & $0.000[0.000]$ & $0.016 * *$ & N/A & N/A \\
\hline Lymphocytes/ $\mu \mathrm{L}$ & 27 & $1.034[0.075]$ & 25 & $0.901[0.081]$ & 11 & $1.307[0.158]$ & 36 & $1.575[0.600]$ & 0.235 & $0.085^{*}$ & 0.440 \\
\hline $\mathrm{CRP}, \mathrm{mg} / \mathrm{dL}$ & 27 & 8.604 [0.943] & 26 & 14.085 [2.197] & 13 & 8.569 [2.353] & 36 & $13.081[1.242]$ & $0.024 * *$ & 0.987 & $0.009 * *$ \\
\hline Creatinine, $\mathrm{mg} / \mathrm{dL}$ & 27 & 1.429 [0.339] & 26 & & 13 & & 36 & $1.197[0.083]$ & & & 0.455 \\
\hline Bilirubin, $\mathrm{mg} / \mathrm{dL}$ & 27 & $0.537[0.045]$ & 25 & & 13 & $0.622[0.081]$ & 36 & $0.757[0.091]$ & & & $0.054 *$ \\
\hline INR & 23 & $1.131[0.015]$ & 25 & $1.703[0.333]$ & 13 & 1.122 [0.019] & 33 & $1.195[0.023]$ & & 0.710 & $0.035^{* *}$ \\
\hline aPTT & 23 & $1.150[0.037]$ & 25 & $1.414[0.123]$ & 13 & $1.155[0.053]$ & 33 & $1.163[0.032]$ & $0.053^{*}$ & 0.937 & 0.781 \\
\hline $\mathrm{pH}$ & 24 & $7.474[0.007]$ & 23 & $7.462[0.013]$ & 11 & $7.426[0.027]$ & 31 & $7.464[0.008]$ & 0.441 & $0.029 * *$ & 0.381 \\
\hline $\mathrm{pO} 2, \mathrm{mmHg}$ & 25 & 75.840 [9.996] & 23 & $72.639[9.213]$ & 11 & 81.891 [11.115] & 30 & $62.933[3.600]$ & 0.816 & 0.721 & 0.199 \\
\hline $\mathrm{pCO} 2, \mathrm{mmHg}$ & 25 & $30.480[0.813]$ & 23 & 29.870 [1.034] & 11 & $30.273[2.170]$ & 31 & $30.194[0.894]$ & 0.642 & 0.913 & 0.817 \\
\hline $\mathrm{PaO} 2 / \mathrm{FiO} 2$ & 24 & $247.875[16.73]$ & 23 & $185.783[19.63]$ & 11 & $194.091[29.81]$ & 29 & $202.828[16.88]$ & $0.020 * *$ & 0.101 & $0.066^{*}$ \\
\hline
\end{tabular}

Comparison between patients treated with CPAP/NIV only (1) and the other groups of patients: CPAP/NIV and DNI (2); ETI (3); NIV failure and ETI (4)

$N$ number of observations, $C P A P / N I V$ continuous positive airway pressure/non-invasive ventilation, $D N I$ do-not-intubate order, $E T I$ endotracheal intubation, $C R P$ C-reactive protein, INR international normalized ratio, aPTT activated prothrombin time, $\mathrm{PaO} / \mathrm{FiO} 2$ arterial oxygen partial tension/fraction inspired oxygen

The value displayed for $t$ tests are $P$ values. ***,** and $*$ indicate significance at the 1,5 and 10 percent critical level

\section{Discussion}

Although much epidemiological data on COVID-19 have been published in the medical literature, there is still a paucity of information about the clinical course of patients accessing the hospital through the ER. Our study is one of the first that analyzes a cohort of patients admitted to medical wards, subintensive and in intensive care units, with detailed data about the outcome of those treated with respiratory support.

The first finding that deserves attention is the high mortality of patients who were admitted to hospital (30.4\%). This number is higher than those from other series: Wang et al [11] reported a $4.3 \%$ death rate among 138 patients admitted for COVID-19 pneumonia, and Guan et al [2] an astonishingly low $1.4 \%$ mortality on a population of 1099 admitted patients. It must be considered though, that the great majority of patients enrolled in these observational studies, had mild form of disease, and that the data from Wang et al. have been calculated when only $41 \%$ of patients had either died or been discharged. Among the 173 patients from the Guan's study who had a severe form of the disease, a composite end-point of death, ICU admission and invasive mechanical ventilation reached the percentage of $24.9 \%$. Differences in death rate could also be secondary to age difference (a median of 64 years in our study vs. 56 and 47 , respectively), and to our stricter criteria for hospital admission. In our series, even patients admitted to ICU and invasively ventilated showed high overall mortality (55.1\%). This percentage does not differ so much from those of Yang et al. [6] who reported a mortality of $61.5 \%$ on 52 critically ill patients, and of Arentz et al. [7] who had $15(68,2 \%)$ deaths and 4 patients, who remained critically ill among 22 severe respiratory patients admitted to their ICU. Report from a cohort of 1591 patients admitted to the ICUs of the Lombardy Region shows a lower mortality rate of $26 \%$; this finding should be questioned because death rate was calculated on the totality of admitted patients, when only $41 \%$ of patients had concluded their hospital course. Indeed, among the 661 patients who died or were discharged from 
ICU, the death toll rises to $61.2 \%$ [5]. Altogether these data give evidence of the dire outcome of patients with the most critical forms of CoV-SARS-2 pneumonia. In our series, all the deceased patients died because of respiratory failure secondary to COVID-19 pneumonia or ARDS. However, the most severe cases, characterized by long ICU admission, were complicated by septic shock, bacterial super-infection, multi-organ failure (MOF) or ventilator-induced lung injury.

A second relevant aspect is related to risk factors. Although our numbers are limited, we confirmed a worse prognosis for older age classes as well as for patients with obesity, diabetes, heart disease or chronic kidney disease.

Conversely, we could not find any association between chronic therapy with ACEI or ARB and mortality, after adjusting for age and hypertension, in accordance with published data $[12,13]$.

With respect to laboratory tests, patients with a $\mathrm{CRP}>10 \mathrm{mg} / \mathrm{dl}$ had worse prognosis than those with lower values.

CT scan of the chest was performed in 264 of 310 patients. Ground grass opacities, crazy paving or consolidations were found in $97.7 \%$ of the tested patients, even in those with no respiratory symptoms. Bedside chest ultrasound demonstrated high concordance compared with chest $\mathrm{CT}$ for the identification of interstitial pneumonia. It should be noted that this achievement has been obtained during a pandemic outbreak, where a high index of suspicion supports the correlation between echographic patterns and clinical presentation. The reliability of ultrasound may be of relevance when ER is overwhelmed by a high number of patients who need to be evaluated over a short period of time $[14,15]$, or in clinical settings where CT is not available. On the other hand, chest X-ray showed a poor performance for the diagnosis of COVID-19 patients. This finding is consistent with clinical literature [16].

Controversy exists in the literature about the effectiveness of, and harm from CPAP and NIV, as poor effectiveness and risk of harm have been shown in other forms of acute respiratory distress syndromes (ARDS) [17-20]. Namendys discourages the use of non-invasive ventilation for critical patients with COVID-19, because he believes, that, according to published data, there is no evidence that it could change the fatal course of the disease $[6,17]$. He also raises concerns about the safety for health workers, due to the risk of spreading virus via aerosol. Gattinoni et al. suggested two clinical entities at the extremity of a continuum: type $\mathrm{L}$, characterized by high lung compliance and prevalence of interstitial ground glass patterns at CT scan, and type $\mathrm{H}$, characterized by low compliance and increased number of consolidations. Type L patients usually present elevated respiratory drive and vigorous respiratory effort, with consequent increase in pulmonary transvascular pressure. Elevated transvascular pressure, together with endothelial damage, is thought to be the cause of the socalled patient self-induced lung injury (PSILI), that can lead to alveolar and interstitial edema. Oxygen administration alone or in association with PEEP or pressure support, can reduce the respiratory effort and prevent the lung injury. If the disease progresses to type $\mathrm{H}$, endotracheal intubation represents the only option. However, the administration of elevated PEEP on a rigid lung, with reduced size of wellaerated lung ("baby lung"), can easily cause ventilatorinduced lung injury (VILI) [21].

Interesting suggestions come from our data on oxygen delivery and ventilation. We were the first to differentiate patients who received a DNI order and were therefore not suitable for therapeutic upgrade. More than half of our patients received only oxygen support, and about $30 \%$ were supported with CPAP or NIV. Considering the latter group as a whole, roughly one-third received CPAP/NIV as an upper therapeutic threshold, after a DNI order was issued. Unfortunately, most of these patients died (88.9\%). Another third of the patients, characterized by younger age, few comorbidities and a less severe degree of respiratory insufficiency, were successfully treated with CPAP/NIV and were discharged home (only 1 death out of 27 patients). Thirty-six patients out of sixty-three underwent endotracheal intubation (ETI) after CPAP/NIV failure, usually within one day of starting ventilator support. Death rate among patients intubated after CPAP/NIV failure was $57.1 \%$ (36 patients out of 63), with mortality rate of $47.2 \%$. This figure is consistent with numbers reported in medical literature for ARDS of any cause [22].

It can be speculated that a significant number of COVID19 patients, younger and with less severe respiratory failure, may benefit from CPAP/NIV without needing tracheal intubation. The use of CPAP/NIV for patients who have a DNI order, can possibly give relief from lack of oxygen, but it does not improve the otherwise poor outcome of the most severe cases. Elevated mortality among intubated patients, together with long clinical course in ICU, represents a crucial question that needs to be answered.

Larger and randomized studies will be needed to better identify patients who could benefit from CPAP/NIV, from those that should be expeditiously intubated. It would also be of interest to investigate whether the start time of CPAP/ NIV has any relevance on the outcome.

Most of the patients of our series were treated with lopinavir-ritonavir and hydroxychloroquine, but neither drug showed an association with outcome. Indeed, at least one randomized clinical trial, published after we started data retrieving, showed no efficacy for lopinavir-ritonavir. The use of hydroxychloroquine, that was initially supported by studies with several limitations [23], has been subsequently questioned by more solid data [24-26] The efficacy of immunosuppressive drugs like steroids and tocilizumab 
is still debated. Their use has a rationale during the hyperinflammation phase characterized by the cytokine storm but conclusive data based on large trial are still needed [27-31].

Our study has several limitations. First of all, management and treatment of patients did not follow a strict protocol, so outcomes may be due to variables other than those that have been controlled. This can be of relevance for patient treated with CPAP/NIV or mechanical ventilation, because we did not record ventilator parameters or the use of prone positioning strategy. Second, many patients that presented clinical features and imaging compatible with COVID-19 were excluded after negative RT-PCR swab. Considering the low sensibility of the RT-PCR assay [10], it is likely that we excluded a considerable percentage of patients with SARS-Cov-2 related interstitial pneumonia. Third, there are some missing data, in particular for obesity and smoking habit. Since individuals with missing information might not be selected at random, this could possibly introduce bias in our results. In particular, we find that individuals whose information about obesity is non-missing are significantly younger and less likely to present pre-existing cardiac and vascular conditions than individual with missing information about obesity. We do not find significant differences (at the 5\% level) in any other observable characteristic between patients with and without missing information for smoking or obesity. Fourth, the study was conducted at the early stage of the pandemic outbreak, when the exact physiopathology of the disease was unknown and there was lack of experience in treating COVID-19 pneumonia. It is possible that the outcome of the patients would have improved over the next weeks. Finally, the decision of excluding DNI patients from intubation and therapeutic up-grade was not based on standardized and clear protocols but on clinical judgment. The arbitrariness of such a complex decision, could have affected the outcome of the most severe cases. In particular, some patients in DNI subgroup could have benefited from therapeutic upgrade reducing the overall mortality.

\section{Conclusions}

This single-center study at Milan, Italy, provides detailed clinical data on 310 patients, admitted to Emergency Room. As a specific point of interest, our data confirm the high mortality of patients that need invasive or non-invasive ventilator support, but also show that in a significant subset of patients with SARS-CoV-2 pneumonia, CPAP/NIV may prevent intubation and can be associated with a favorable outcome.
Funding No funding has been received.

Data availability Data are available for further request.

\section{Compliance with ethical standards}

Conflict of interest The authors declare no conflict of interest.

Statement of Human and animal rights The study was conducted in accordance with the ethical standards of the responsible committee on human experimentation of the institutional review board at Grande Ospedale Metropolitano Niguarda. Non animals were involved in the study.

Informed consent Written informed consent was waived by the ethics commission of the designated hospital, in accordance with governmental regulations on observational retrospective studies.

\section{References}

1. Italian Institute for International Political Studies. Covid-19 and Italy's case fatality rate: what's the catch? https://www.ispionline .it/en/publication/covid-19-and-italys-case-fatality-rate-whats -catch-25586. Accessed Apr 132020

2. Guan WJ, Ni ZY, Hu Y et al (2020) China Medical Treatment Expert Group for Covid-19. Clinical characteristics of coronavirus disease 2019 in China. N Engl J Med 382(18):1708-1720. https:// doi.org/10.1056/NEJMoa2002032

3. Huang C, Wang Y, Li X et al (2020) Clinical features of patients infected with 2019 novel coronavirus in Wuhan, China. Lancet. https://doi.org/10.1016/S0140-6736(20)30183-5

4. Remuzzi A, Remuzzi G (2020) COVID-19 and Italy: what next? Lancet. https://doi.org/10.1016/S0140-6736(20)30627-9

5. Grasselli G, Zangrillo A, Zanella A et al (2020) Baseline characteristics and outcomes of 1591 patients infected with SARSCoV-2 admitted to ICUs of the Lombardy region, Italy. JAMA. https://doi.org/10.1001/jama.2020.5394

6. Yang X, Yu Y, Xu J et al (2020) Clinical course and outcomes of critically ill patients with SARS-CoV-2 pneumonia in Wuhan, China: a single-centered, retrospective, observational study. Lancet Respir Med. https://doi.org/10.1016/S2213-2600(20)30079-5

7. Arentz M, YimE KL et al (2020) Characteristics and outcomes of 21 critically ill patients with COVID-19 in Washington State. JAMA. https://doi.org/10.1001/jama.2020.4326

8. Zhou F, Yu T, Du R et al (2020) Clinical course and risk factors for mortality of adult inpatients with COVID-19 in Wuhan, China: a retrospective cohort study. Lancet 395(10229):1054-1062. https ://doi.org/10.1016/S0140-6736(20)30566-3

9. WHO (2020) Clinical management of severe acute respiratory infection when novel coronavirus $(\mathrm{nCoV})$ infection is suspected: interim guidance, 25 January 2020. Accessed Mar 30 2020. https ://apps.who.int/iris/handle/10665/330854

10. Wang W, Xu Y, Gao R, Lu R, Han K et al (2020) Detection of SARS-CoV-2 in different types of clinical specimens. JAMA. https://doi.org/10.1001/jama.2020.3786

11. Wang D, Hu B, Hu C et al (2020) Clinical characteristics of 138 hospitalized patients with 2019 novel Coronavirus-infected pneumonia in Wuhan, China. JAMA 323(11):1061-1069. https://doi. org/10.1001/jama.2020.1585 
12. Gao C, Cai Y, Zhang K et al (2020) Association of hypertension and antihypertensive treatment with COVID-19 mortality: a retrospective observational study. Eur Heart J 41:2058-2066

13. Gnavi R, Demaria M, Picariello R et al (2020) Therapy with agents acting on the renin-angiotensin system and risk of SARSCoV-2 infection. Clin Infect Dis. https://doi.org/10.1093/cid/ciaa6 34

14. Peng QY, Wang XT, Zhang LN (2020) Findings of lung ultrasonography of novel corona virus pneumonia during the 20192020 epidemic. Intensive Care Med. https://doi.org/10.1007/s0013 4-020-05996-6

15. Buonsenso D, Chiaretti A (2020) COVID-19 outbreak: less stethoscope, more ultrasound. Lancet Respir Med. https://doi. org/10.1016/S2213-2600(20)30120-X

16. Fang Y, Zhang H, Xie J et al (2020) Sensitivity of chest CT for COVID-19: comparison to RT-PCR. Radiology. https://doi. org/10.1148/radiol.2020200432

17. Namendis-Silva SA (2020) Respiratory support for patients with COVID-19 infection. Lancet Respir Med. https://doi.org/10.1016/ S2213-2600(20)30110-7

18. Bellani G, Laffey JG, Pham T, Madotto F, Fan E et al (2017) Noninvasive ventilation of patients with acute respiratory distress syndrome: insights from the LUNG SAFE study. Am J Respir Crit Care Med 195(1):67-77

19. He H, Sun B, Liang L, Li Y, Wang H et al (2019) A multicenter RCT of noninvasive ventilation in pneumonia-induced early mild acute respiratory distress syndrome. Crit Care 23:300. https://doi. org/10.1186/s13054-019-2575-6

20. Masclans JR, Perez M, Almirall J, Lorente L, Marques A et al (2013) Early non-invasive ventilation treatment for severe influenza pneumonia. Clin Microbiol Infect 19:249-256

21. Marini JJ, Gattinoni L (2020) Management of COVID-19 Respiratory Distress. JAMA 323(22):2329-2330

22. Bellani G, Laffey JG, Pham T et al (2017) Noninvasive ventilation of patients with acute respiratory distress syndrome. Insights from the LUNG SAFE study. Am J Respir Crit Care Med 195(1):67-77. https://doi.org/10.1164/rccm.201606-1306OC

23. Gautret P, Lagier JC, Parola P, Meddeb L, Mailhe M et al (2020) Hydroxychloroquine and azithromycin as a treatment of
COVID-19: results of an open-label non-randomized clinical trial. Int J Antimicrob Agents 56(1):105949

24. Shah S, Das S, Jain A, Misra DP, Negi VS (2020) A systematic review of the prophylactic role of chloroquine and hydroxychloroquine in coronavirus disease-19 (COVID-19). Int J Rheum Dis 23(5):613-619. https://doi.org/10.1111/1756-185X.13842

25. Molina JM, Delaugerre C, Le Goff J et al (2020) No evidence of rapid antiviral clearance or clinical benefit with the combination of hydroxychloroquine and azithromycin in patients with severe COVID-19 infection. Med Mal Infect 50(4):384. https:// doi.org/10.1016/j.medmal.2020.03.006

26. Cavalcanti AB, Zampieri FG, Rosa RG et al (2020) Hydroxychloroquine with or without azithromycin in mild-to-moderate Covid-19. N Engl J Med. https://doi.org/10.1056/NEJMoa2019 014

27. Arabi YM, Mandourah Y, Al-Hameed F et al (2018) Corticosteroid therapy for critically ill patients with middle east respiratory syndrome. Am J Respir Crit Care Med 197:757-767

28. Xu X, Han M, Li T et al (2020) Effective treatment of severe COVID-19 patients with tocilizumab. Proc Natl Acad Sci U S A 117(20):10970-10975. https://doi.org/10.1073/pnas.2005615117

29. Mehta P, McAuley DF, Brown M et al (2020) COVID-19: consider cytokine storm syndromes and immunosuppression. Lancet 395(10229):1033-1034. https://doi.org/10.1016/S0140 $-6736(20) 30628-0$

30. Zha L, Li S, Pan L et al (2020) Corticosteroid treatment of patients with coronavirus disease 2019 (COVID-19). Med J Aust 212(9):416-420. https://doi.org/10.5694/mja2.50577

31. Wang Y, Jiang W, He Q, Wang C, Wang B, Zhou P et al (2020) Early, low-dose and short-term application of corticosteroid treatment in patients with severe COVID-19 pneumonia: singlecenter experience from Wuhan, China. medRxiv. https://doi. org/10.1101/2020.03.06.20032342

Publisher's Note Springer Nature remains neutral with regard to jurisdictional claims in published maps and institutional affiliations. 